\title{
Evaluación de dos tratamientos psicológicos para mujeres embarazadas con sintomatología ansiosa ${ }^{1}$
}

\author{
Evaluation of two psychological treatments \\ for pregnant women with anxious symptomatology
}

\author{
Carlos Augusto Rodríguez Durán y Ana Beatriz Moreno Coutiño
}

\begin{abstract}
Citación: Rodríguez D., C.A. y Moreno C., A.B. (2021). Evaluación de dos tratamientos psicológicos para mujeres embarazadas con sintomatología ansiosa. Psicología y Salud, 31(1), 69-79. https:// doi.org/10.25009/pys.v31i1.2677.
\end{abstract}

\section{RESUMEN}

\begin{abstract}
Introducción: La sintomatología ansiosa en mujeres embarazadas tiene una prevalencia de entre 15 y $50 \%$ y puede provocar complicaciones en este periodo. Los programas basados en la atención plena y el tratamiento cognitivo-conductual son dispositivos terapéuticos eficaces para combatir esta problemática. Objetivo: En un hospital de la Ciudad de México, evaluar el efecto de dos intervenciones psicológicas para reducir los síntomas de ansiedad en mujeres embarazadas: atención plena compasiva y terapia cognitivo-conductual, de ocho sesiones individuales cada intervención y con una duración de 90 minutos cada módulo. Método: Se implementó un diseño intrasujetos con evaluaciones en el pretratamiento, el postratamiento y el seguimiento de tres meses. La asignación de las participantes a cada tratamiento fue aleatoria. Seis participantes finalizaron su tratamiento y los seguimientos. Se utilizaron como instrumentos psicométricos un inventario de ansiedad, una escala de atención plena y una escala de compasión. Resultados: Se calculó el índice de Cambio Clínico Objetivo de Cardiel. Ambos tratamientos fueron eficaces para reducir la sintomatología ansiosa en mujeres embarazadas. Las participantes que asistieron al modelo de atención plena compasiva lograron aumentar sus niveles de atención plena y de compasión. Discusión: Una limitación del estudio fue el pequeño número de participantes. Se sugiere que estos modelos continúen siendo probados en el ámbito hospitalario para recabar más evidencia sobre estos tratamientos psicológicos.
\end{abstract}

Palabras clave: Ansiedad; Embarazo; Terapia cognitivo-conductual; Atención plena; Compasión.

\begin{abstract}
Introduction: Anxious symptomatology in pregnant women affects between $15 \%$ and up to $50 \%$ of them and can cause obstetric complications in this period. Programs based on mindfulness and cognitive behavioral therapies have shown to be effective therapeutic devices to cope with this problem. Objective: To evaluate the effects of two psychological interventions for the reduction of anxiety symptoms in pregnant women: a) compassionate mindfulness and b) cognitive behavioral therapy, with participants randomly assigned to each treatment. Treatments were given through eight individual sessions lasting 90 minutes per module. Method: An anxiety inventory, a mindfulness scale, and a compassion scale were used as measurement instruments through a within-subject design with evaluations at pretreatment, posttreatment and a 3-month follow-up. Six participants completed treatments and follow-ups. Results: Cardiel's clinical objective change formula on data revealed that both treatments were effective in reducing anxious symptoms in pregnant women. Participants exposed to the compassionate mindfulness model increased their levels of mindfulness
\end{abstract}

\footnotetext{
${ }^{1}$ El autor con Cvu: 547237, número del becario: 298881, y número de apoyo: 449439, agradece al Consejo Nacional de Ciencia y Tecnología (CONACYT) por el financiamiento para este trabajo.

${ }^{2}$ Facultad de Psicología, Universidad Nacional Autónoma de México, Av. Universidad 3004, Col. Copilco Universidad, Coyoacán, Ciudad de México, México. Correos electrónicos: carlosreduran@hotmail.com y moca99_99@yahoo.com. Artículo recibido el 3 de octubre de 2019 y aceptado el 16 de abril de 2020 .
} 
and compassion. Discussion: As a limitation, in this study the number of participants was small. It is suggested that these models continue to be tested in hospital settings to gather further evidence regarding these psychological treatments.

Keywords: Anxiety; Pregnancy; Cognitivebehavioral therapy; Mindfulness; Compassion.

\section{INTRODUCCIÓN}

S egún la Organización Mundial de la Salud, una de cada trece personas en el mundo sufre de ansiedad, y sus trastornos más comunes son las fobias específicas y las sociales. Estos trastornos se desarrollan a partir de un complejo conjunto de factores de riesgo, los cuales incluyen la genética, la química del cerebro, la personalidad y los eventos de vida (World Health Organization, 2020). La ansiedad es pues un problema de salud pública, por lo que se considera importante desarrollar intervenciones psicosociales que puedan resolver eficazmente este padecimiento (Clark y Beck, 2013).

La ansiedad se puede definir como la percepción psicológica de una amenaza futura, y se manifiesta fisiológica, afectiva, cognitiva y conductualmente (Rygh y Sanderson, 2004). Es también un estado de activación del sistema nervioso central y del sistema nervioso autónomo que prepara al organismo para afrontar situaciones de peligro (Barrondo, Martínez y González, 2008).

Internacionalmente, la prevalencia de la ansiedad en mujeres embarazadas se encuentra en un rango que va de entre 15 y 30\% (Fairbrother, Janssen, Antony, Tucker y Young, 2016; Silva, Nogueira, Clapis y Leite, 2017). En México, un estudio descriptivo estimó esa prevalencia en 50.5\% de las pacientes embarazadas, derechohabientes del Instituto Mexicano del Seguro Social, donde se estudió a 103 mediante la Escala de Ansiedad de Goldberg (Aceves, Ureña, Contreras, Magaña y Romero, 2013).

La ansiedad conlleva respuestas psicológicas como la percepción de temor, intranquilidad, angustia y preocupación (Cano, Dongil, Salguero y Wood, 2013), así como un aumento de la tensión muscular, respiración, presión arterial y frecuencia cardiaca (Hoehn-Saric, 1998). Algunas investigaciones señalan que cuando esta sintomatología aparece en el transcurso del embarazo, se asocia con complicaciones obstétricas (Leonetti y Martins, 2007), bajo peso del producto al nacer, nacimiento prematuro (Ding et al., 2014), mayor duración del parto (Reck et al., 2013) y afectaciones en el neurodesarrollo fetal (Glover, Ahmed-Salim y Capron, 2016).

Por lo anterior, se considera primordial atender a las mujeres embarazadas que exhiban una sintomatología ansiosa mediante tratamientos psicológicos basados en evidencia, a fin de prevenir el desarrollo de problemas de ansiedad y reducir los riesgos en la salud de la madre y el bebé (Lemon, Vanderkruik y Dimidjian, 2015).

\section{Terapia cognitivo-conductual}

La terapia cognitivo-conductual (TCC en lo sucesivo) es una forma de psicoterapia empíricamente validada que ha demostrado ser efectiva en diferentes trastornos psiquiátricos, como la depresión y la ansiedad, así como en los de personalidad (Beck, 2005). De acuerdo con Kingdon y Dimech (2008), se puede aplicar de forma individual o grupal y es efectiva en diferentes grupos de edad, en la comparación directa con otras intervenciones y en el mantenimiento de beneficios al término del tratamiento.

La TCC se ha evaluado como una intervención psicológica para una amplia gama de trastornos de ansiedad (Díaz, Ruiz y Villalobos, 2017). Incluye la combinación de los siguientes componentes: psicoeducación, automonitoreo, reestructuración cognitiva y técnicas de regulación fisiológica, como la relajación muscular progresiva y la respiración profunda (Clark y Beck, 2013). Asimismo, se ha reportado que la TCC y la farmacoterapia son igualmente eficaces para el tratamiento de corto plazo de los trastornos de ansiedad; sin embargo, la primera se percibe como menos aversiva para los participantes, pues las tasas de abandono son menores y se reduce el riesgo de recaídas en comparación con la farmacoterapia sola (Epp, Dobson y Cottraux, 2009). 
Una revisión sistemática de los tratamientos farmacológicos y no farmacológicos para las mujeres embarazadas diagnosticadas con trastornos de ansiedad (Marchesi et al., 2016) sugiere que la TCC debiera ofrecerse a estas como primera opción debido a su eficacia para tratarlos.

Un estudio piloto examinó la efectividad de la TCC grupal en diez mujeres diagnosticadas con ansiedad en su etapa perinatal. Las participantes completaron seis sesiones semanales con duración de dos horas cada una. Como resultado hubo una reducción estadísticamente significativa de la sintomatología ansiosa y depresiva al término del tratamiento. Aunado a lo anterior, las participantes reportaron una mayor aceptación y complacencia con la intervención. Estos hallazgos sugirieron que la TCC es eficaz para disminuir la ansiedad en mujeres embarazadas (Green, Haber, Frey y McCabe, 2015).

\section{Atención plena y compasión}

La atención plena es una alternativa psicoterapéutica que forma parte de las terapias cognitivo-conductuales de tercera generación, formalmente definida por Hayes (2004) en la revista Behavior Therapy, con lo que se implantó una nueva propuesta enriquecida que trae mejoras en la atención a la salud (Pérez, 2012). El entrenamiento en atención plena es una práctica milenaria conservada a lo largo de generaciones entre los budistas, aunque en las últimas décadas ha sido conceptualizada como una habilidad psicológica, de manera que se ha implantado en contextos clínicos hospitalarios. De hecho, en numerosos países se ha integrado a la mayor parte de los modelos psicoterapéuticos actuales (Miró, 2012).

Atención plena es la traducción de la palabra inglesa mindfulness, la cual proviene a su vez de sati, una palabra pali, un idioma índico o prácrito, que se puede traducir como "estar atento", "mantenerse consciente", "tener conciencia plena" o "presencia plena", la cual se refiere a que la persona se concentra en la tarea que está realizando en ese momento sin que la mente divague sobre el futuro o el pasado, y sin sensación de apego o rechazo, lo que le genera energía, claridad y alegría (Brown y Ryan, 2003).
La práctica de la atención plena no estaría completa si se deja de lado el cultivo de la compasión, término que se deriva del sánscrito karuna y que entraña un sincero deseo de aliviar el sufrimiento propio y el de los demás, así como la acción que se pone en práctica para conseguirlo (Simón y Germer, 2011). La definición de la compasión está integrada por dos partes: una motivacional, que implica la aspiración verdadera que abarca a todo ser, incluido uno mismo, por lo que no se trata de sacrificarse a costa del bienestar de los demás; la segunda parte, que involucra las acciones concretas que se emprenden, que son motivadas por la aspiración compasiva y que formarán parte de las causas que implantarán un cambio en la experiencia propia y en la de los demás (Hangartner, 2013). La compasión, al ser una parte fundamental de la filosofía que sustenta la atención plena, puede ser entrenada en cualquier persona con la ayuda de prácticas meditativas (Bornemann y Singer, 2013).

Asimismo, los tratamientos basados en atención plena mejoran la salud mental al incidir en variables tales como la regulación emocional, el estrés y la sintomatología ansiosa. Estos resultados se han encontrado en diversos grupos etarios (adultos y de tercera edad), así como en personas con trastorno de ansiedad generalizada, de pánico y de ansiedad social (Goldin y Gross, 2010; Helmes y Ward, 2017; Hoffman et al., 2012; Koszycki et al., 2016).

Recientes estudios clínicos experimentales sugieren que la práctica de la atención plena en mujeres embarazadas disminuye los niveles de estrés, ansiedad y depresión, al mismo tiempo que aumenta los estados afectivos positivos. Las participantes han reportado buena adherencia a este modelo de intervención. Al disminuir el número de nacimientos complicados, la atención plena puede reducir la duración de la hospitalización sin generar gastos extras para la paciente ni para el hospital; otra ventaja es que dicha intervención puede llevarse a cabo individual o grupalmente (Goodman et al., 2014; Guardino, Dunkel- Schetter, Bower, Lu y Smalley, 2014; Matvienko-Sikar, Lee, Murphy y Murphy, 2016; O’Leary, Dockray y Hammond, 2016). 
En específico, las intervenciones basadas en la atención plena que integran componentes centrados en la autocompasión también han reportado resultados prometedores para el tratamiento de sintomatología ansiosa en las mujeres embarazadas (Pereira et al., 2016).

Aunque la ansiedad durante el embarazo es común y puede causar problemas de salud física y mental en la futura madre, así como en el desarrollo del producto, se ha prestado poca atención a este importante problema, por lo que hay escasas investigaciones y se ha dejado a las mujeres embarazadas con pocas opciones de tratamientos psicológicos basados en evidencia (Lemon et al., 2015). De acuerdo a lo anterior, es importante que las mujeres embarazadas de nuestro país cuenten con servicios de salud psicológica enfocados específicamente a la reducción de la ansiedad en esa etapa (Pimentel, Morales, Sánchez y Meza, 2013).

Por consiguiente, el objetivo del presente análisis fue probar la eficacia de dos tratamientos psicológicos para mujeres embarazadas con sintomatología ansiosa mediante comparaciones intrasujetos, llevándose a cabo un estudio piloto para conocer el efecto de los modelos de intervención basados en la atención plena compasiva (APC en lo sucesivo) y la TCC en mujeres embarazadas en un hospital general de la Ciudad de México.

\section{MÉTODO}

\section{Participantes}

En el área de consulta externa del hospital, se invitó a cuarenta mujeres embarazadas a participar en un curso-taller y realizar una evaluación inicial. El principal criterio de inclusión fue que presentaran sintomatología ansiosa en los siguientes niveles: algo, bastante o mucha ansiedad; no se aceptaron mujeres que estuvieran bajo tratamiento psiquiátrico. En total, dieciocho mujeres que cumplieron los criterios de inclusión completaron la preevaluación, por lo que fueron asignadas mediante un método aleatorio simple a los dos grupos de tratamiento (nueve al modelo APC y nueve al de TCC). Seis participantes (tres en APC y tres en TCC) concluyeron los ocho módulos de estas intervenciones, realizaron la evaluación tras el tratamiento y se sometieron al seguimiento a los tres meses.

Las razones por las cuales las restantes mujeres no continuaron en el estudio fueron las siguientes: horarios de trabajo inflexibles, largas distancias que dificultaban llegar a tiempo a las sesiones, cambio de hospital para continuar sus controles médicos y problemas económicos. En la Tabla 1 se resumen los datos sociodemográficos de las seis participantes que concluyeron el tratamiento.

Tabla 1. Datos sociodemográficos de las participantes $n=6$.

\begin{tabular}{|c|c|c|c|c|c|}
\hline Participante & Edad & Estado civil & Escolaridad & $\begin{array}{c}\text { Edad } \\
\text { gestacional }\end{array}$ & $\begin{array}{c}\text { Enfermedad } \\
\text { psiquiátrica }\end{array}$ \\
\hline $1 \mathrm{TCC}$ & 39 & Unión libre & Licenciatura & 21 semanas & No \\
\hline $2 \mathrm{TCC}$ & 34 & Soltera & Licenciatura & 24 semanas & No \\
\hline $3 \mathrm{TCC}$ & 37 & Soltera & Licenciatura & 24 semanas & No \\
\hline $1 \mathrm{APC}$ & 32 & Casada & Preparatoria & 21 semanas & No \\
\hline $2 \mathrm{APC}$ & 36 & Casada & Doctorado & 22 semanas & No \\
\hline $3 \mathrm{APC}$ & 25 & Soltera & Licenciatura & 23 semanas & No \\
\hline
\end{tabular}

\section{Diseño}

El diseño empleado en el estudio fue intrasujetos, con medidas repetidas de las variables dependientes en pretratamiento, postratamiento y seguimiento (Barlow, 2009; Hernández, Fernández y Baptista, 2010).

\section{Instrumentos}

Inventario Mexicano de Ansiedad, Depresión e Ideación Suicida, versión Beta (IMADIS) (Jurado, en prensa).

Este instrumento cuenta con un valor de confiabilidad medida por el coeficiente $\alpha$ de Cronbach de 
0.96, y validez concurrente con el Inventario de Ansiedad de Beck $(r=.70)$. Está constituido por 21 reactivos con opciones de respuesta que van de 1, "Nunca", a 5, "Siempre".

Escala de Atención Plena (MAAS) (Brown y Ryan, 2003).

Adaptada y validada para población mexicana (por López et al., 2015), esta escala tiene un coeficiente $\alpha$ de Cronbach de .89 y validez concurrente con el Cuestionario de Cinco Facetas de la Atención Plena $(r=.45)$ (Baer, 2011). Cuenta con quince reactivos con opciones de respuesta que van de 1 , "Casi siempre", a 6, "Casi nunca".

Escala de Compasión (ECOM) para Población Mexicana (Tello y Coutiño, 2019).

Este instrumento tiene un coeficiente $\alpha$ de Cronbach de .90 y una validez estructural. Los indicadores de bondad de ajuste presentan niveles adecuados. La escala consta de 17 reactivos con opciones de respuesta que van de 1, "Nunca", a 7 , "Siempre".

\section{Procedimiento}

Se reclutó a mujeres embarazadas que asistían al servicio de consulta externa del hospital. Por medio de una invitación personal en forma de tríptico, se les explicó el objetivo, la duración y los beneficios de participar en los tratamientos psicológicos. A quienes estuvieron interesadas se les citó a una sesión de evaluación mediante un pase que contenía los siguientes datos: nombre y teléfono del psicólogo, así como día y fecha de la consulta. Los procedimientos de evaluación e intervención se llevaron a cabo en un aula ubicada dentro del hospital, lugar que contaba con iluminación adecuada, dos sillas, una mesa y los materiales para llevar a cabo las intervenciones (pantalla con bocinas y computadora), asegurándose así que todas las participantes fueran expuestas a las mismas condiciones ambientales.

Las actividades realizadas en la sesión de evaluación fueron las siguientes: medición de los signos vitales al inicio y al final de la sesión, aplicación de entrevista inicial semiestructurada, aplicación de los instrumentos psicométricos señalados antes, evaluación de los criterios de inclusión, firma de una carta de consentimiento informado, evaluado por el Comité de Ética del hospital, y asignación aleatoria de cada participante a los tratamientos psicológicos (TCC o APC). La duración promedio de estas sesiones fue de 90 minutos.

Los tratamientos se implementaron respectivamente en ocho módulos, con duración aproximada de 90 minutos. Un psicólogo con entrenamiento tanto en APC como en TCC, y teniendo un rol directivo, hizo ambas intervenciones, para esto llevó a cabo exposiciones teóricas y ejercicios prácticos. La integridad del tratamiento fue evaluada por medio de registros efectuados por una psicóloga observadora independiente, quien calificó cada sesión mediante una lista cotejable de actividades a realizar por parte del terapeuta. En la psicoeducación, ambos tratamientos utilizaron los componentes de ansiedad en el embarazo y desregulación emocional que a continuación se describen:

a) Ansiedad y embarazo: Se explicaron los factores que inciden en la ansiedad (Sierra, Ortega y Zubeidat 2003), la frecuencia de la ansiedad en el embarazo (Aceves et al., 2013), la asociación entre sintomatología ansiosa elevada y las complicaciones obstétricas (Leonetti y Martins, 2007), los daños provocados por la ansiedad en el desarrollo fetal (Ding et al., 2014) y el desequilibrio fisiológico (Reck et al., 2013).

b) Desregulación emocional: Se explicó asimismo cómo se relaciona la mente con el cuerpo y que la respuesta fisiológica del miedo genera un desequilibrio en las funciones de los sistemas nerviosos central y autónomo (eje simpático-medular-adrenal y eje hipotalámico-hipofisario-adrenal), lo que provoca procesos de estrés y ansiedad en las personas y, sobre todo, afecta la salud de las mujeres embarazadas y del producto (Barrondo et al., 2008; Leonetti y Martins, 2007; Reck et al., 2013).

\section{Modelo de la APC para mujeres embarazadas con sintomatología ansiosa}

\section{Introducción al modelo de atención plena compasiva.}

Definición y beneficios de la atención plena compasiva: Se explicó que la atención plena compasiva es la capacidad de permanecer en el momento 
presente, sin juzgar, con aceptación, sin generar aversión o apego a los eventos mentales, y con el reconocimiento y aspiración de aliviar el sufrimiento propio y el de los demás (Paquini y Coutiño, 2017).

\section{Atención plena.}

a) Entrenamiento en el entendimiento de la causalidad. Mediante ejemplos y preguntas de reflexión, se abordó la causalidad y la responsabilidad de los actos; se explicó que a cada acción (ya sea un pensamiento, palabra o conducta) le corresponde una reacción.

b) Entrenamiento en la percepción de la interdependencia: Se señaló que la interdependencia es la característica de la realidad que afirma la codependencia y la interrelación de todos los fenómenos.

c) Entrenamiento en la percepción de la impermanencia: Se explicó que la impermanencia es una de las características de la realidad, ya que todos los fenómenos compuestos se encuentran en constante cambio.

d) Entrenamiento en el reconocimiento de la vacuidad: Se expuso que cada fenómeno compuesto, incluyendo pensamientos, palabras y acciones, carece de existencia inherente.

\section{Compasión.}

a) Sufrimiento: Se detalló que el sufrimiento es una condición presente en la vida de todas las personas. La principal causa del sufrimiento es el apego, así como la aversión y la ignorancia, o el no saber cómo aliviar nuestro sufrimiento.

b) Compasión: Se señaló que la compasión es un sincero deseo de aliviar nuestro sufrimiento y el del prójimo, y se describieron las acciones que se ponen en práctica para conseguirlo (Simón y Germer, 2011).

c) Acciones virtuosas: Se expuso que la atención plena y la compasión son un equilibrio que permite practicar virtudes, las cuales se pueden definir como acciones que producen beneficios a algún ser vivo y que nos liberan a nosotros mismos y a los demás del sufrimiento.

\section{Meditación.}

a) Meditación formal en la atención plena: Se entrenó en las técnicas de meditación Shamata, o mente en calma, que se compone de tres habilidades: la postura, la respiración y la motivación adecuada. Cada sensación, pensamiento y emoción se reconocen y aceptan gentilmente, sin juzgarse, y luego se liberan, mientras la atención regresa a la respiración.

b) Meditación formal en la compasión: Se llevó a cabo generando mentalmente la aspiración de liberar a los seres del sufrimiento, para lo cual se utilizaron las técnicas guiadas de amor bondadoso, o Metta Bhavana (Hangartner, 2013), y una meditación de intercambio de uno mismo con los demás, o Tonglen (Ricard, 2009).

c) Meditación informal de la atención plena: Se enseñó a cada participante a dirigir y mantener intencionalmente la atención a la experiencia presente y en situaciones de la vida cotidiana. Se realizaron meditaciones al comer, caminar, lavarse las manos y encender la luz.

d) Meditación informal de la compasión: Se explicó que la compasión correcta implica realizar acciones que alivien el sufrimiento en el momento en el que se detecta. Se practicó el reconocimiento diario del sufrimiento y el anhelo de liberarse de él, generando acciones, palabras o pensamientos que contribuyen a cambiar la experiencia.

\section{Modelo de la TCC para mujeres embarazadas con sintomatología ansiosa}

\section{Introducción al modelo cognitivo-conductual.}

Se explicó el modelo cognitivo-conductual de la experiencia de la ansiedad y preocupación (Borkovec, 2006) abordando los estímulos provocadores de tales respuestas para notar las sensaciones, pensamientos e imágenes asociadas. La experiencia de la ansiedad se divide en el estímulo disparador, y luego en tres diferentes áreas: la cognitiva, la fisiológica y la conductual (Rygh y Sanderson, 2004).

\section{Relajación.}

Se explicó a las participantes que el fin de la relajación es reducir los síntomas fisiológicos de la ansiedad, y de este modo contribuir a la reducción de las preocupaciones. Las técnicas ocupadas fueron las siguientes:

a) Tensión-relajación: Sentadas en una silla, realizaron ejercicios de tensión-relajación en catorce 
grupos musculares, para lo que utilizaron las técnicas de profundización de la relajación mediante la respiración lenta, regular y diafragmática, al final de lo cual se puso en práctica la respiración relajada.

b) Relajación mediante recuerdo: Consiste en concentrarse en los grupos musculares y en liberar la tensión existente en los mismos mediante el recuerdo de las sensaciones de relajación logradas en las prácticas anteriores.

c) Relajación suscitada por señal: Se hacen cuatro o cinco respiraciones lentas, se repite la palabra relajación $\mathrm{u}$ otra similar al espirar, y al hacer esto se libera toda la tensión en el cuerpo, concentrándose la participante en las sensaciones de relajación.

d) Relajación diferencial en posición sentada y de pie: Consiste en tensar solamente aquellos músculos directamente requeridos por una posición o actividad. La tensión de los músculos no requeridos se reduce al máximo. Se hace cuando no se está activo, cuando se está mínimamente activo, y cuando se está claramente activo.

e) Relajación rápida: Se lleva a cabo cuatro veces en las siguientes condiciones: sentada, de pie, no activa y activa. La relajación rápida combina las estrategias de relajación aprendidas en los módulos anteriores. Se practica diez veces al día en las situaciones normales de la vida cotidiana y lleva un registro de la práctica de relajación.

\section{Reestructuración cognitiva.}

a) Primer paso: Se incrementa la conciencia de la actividad cognitiva durante los episodios de preocupación y se emplea un registro de estos, haciéndose una diferenciación entre los hechos concretos y la interpretación de una experiencia. Se reconocen las distorsiones cognitivas más comunes en pensamientos y suposiciones.

b) Segundo paso: Se desafían las distorsiones cognitivas mediante la técnica de descubrimiento guiado a través del método socrático para cuestionar y alterar las distorsiones cognitivas y, por consiguiente, las respuestas emocionales.

c) Tercer paso: Se generan puntos de vista alternativos, como imaginar el evento angustiante desde la perspectiva de una tercera persona, cambiar los roles con alguien que pueda pasar la misma situación, ver el evento en un contexto más amplio, así como imaginar lo peor y lo mejor en un escenario más realista.

d) Cuarto paso: Ante un episodio de ansiedad, se crea una respuesta racional que pueda ser evaluada por experiencias de la vida real. Se enseña a la participante cómo usar un formato de respuesta racional para crear una interpretación más adaptativa del evento y para solidificar el cambio en las cogniciones.

4. Mantenimiento y prevención de recaídas.

a) Prevención de recaídas: Se utilizan las llamadas "metáfora del viaje" y la "montaña del cambio". Se explica la diferencia entre caída y recaída, dejándose como tarea de mantenimiento practicar de modo regular las técnicas aprendidas en las situaciones normales y estresantes de su vida cotidiana.

\section{Postratamiento y seguimiento.}

A cada participante se le hicieron mediciones postratamiento y de seguimientos de tres meses, evaluándose las mismas variables psicológicas del pretratamiento.

\section{Análisis estadístico}

Las variables psicométricas se midieron en escalas de intervalo y se evaluaron mediante el índice de Cambio Clínico Objetivo (CCO) (Cardiel, 1994), el cual compara la diferencia entre la puntuación postest y pretest dividida entre el pretest; indica deterioro o mejoría clínica. Para esta variable se evaluó el pretratamiento, el postratamiento y el seguimiento a los tres meses.

\section{RESULTADOS}

La Figura 1 señala los niveles de sintomatología ansiosa en la escala MAAS, medidos en las evaluaciones de pretratamiento, postratamiento y seguimiento. La gráfica izquierda muestra la disminución de esta variable en tres participantes del modelo de TCC; asimismo, la gráfica derecha muestra la reducción de dicha variable en tres participantes del modelo de APC. Es importante mencionar que, de acuerdo con los indicadores en esta escala (poca ansiedad [1-29], algo de ansiedad [30-34], bastante ansiedad [35-41] y mucha an- 
siedad [42-93]), las participantes pasaron de tener "algo de ansiedad" en el pretratamiento a "poca ansiedad" en el postratamiento y el seguimiento. Finalmente, se puede notar en el seguimiento una mayor tendencia a mantener la reducción de la ansiedad en las participantes que asistieron al programa de APC, en contraste con las participantes del programa de TCC.

Figura 1. Niveles de sintomatología ansiosa.
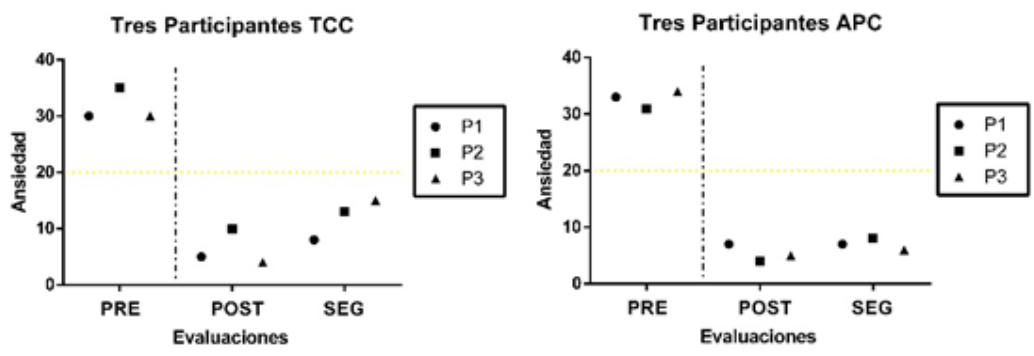

La Tabla 2 describe los porcentajes obtenidos por cada participante, mediante el $\mathrm{CCO}$, en la variable de ansiedad. Las tres participantes que asistieron al modelo de TCC disminuyeron sus niveles de sintomatología ansiosa, y fue así como lograron una mejora clínica significativa en términos de re-

ducción del porcentaje en el postratamiento y en el seguimiento. De igual manera, las tres mujeres en el modelo de APC también obtuvieron una mejora clínica al reducir sus porcentajes de ansiedad de manera significativa.

Tabla 2. CCO en la variable Ansiedad.

\begin{tabular}{|c|c|c|c|}
\hline \multirow{2}{*}{ Tratamiento } & \multirow{2}{*}{ Participantes } & \multicolumn{2}{|c|}{ Ansiedad } \\
\cline { 2 - 4 } & & Post-pre/pre & Seg-pre/pre \\
\hline \multirow{3}{*}{ TCC } & P1 & $-83 \%$ & $-73 \%$ \\
\cline { 2 - 4 } & P2 & $-71 \%$ & $-62 \%$ \\
\cline { 2 - 4 } & P3 & $-86 \%$ & $-50 \%$ \\
\hline \multirow{3}{*}{ APC } & P1 & $-78 \%$ & $-78 \%$ \\
\cline { 2 - 4 } & P2 & $-87 \%$ & $-74 \%$ \\
\cline { 2 - 4 } & P3 & $-85 \%$ & $-82 \%$ \\
\hline
\end{tabular}

En la Figura 2 se grafican los niveles de atención plena de acuerdo con la escala IMADIS en las tres participantes que asistieron al programa de APC. La participante 1 obtuvo 60 puntos en el pretratamiento, aumentó a 79 en el postratamiento y disminuyó a 77 puntos en el seguimiento. La participante 2 alcanzó 66 puntos en el pretratamiento, aumentó a 82 en el postratamiento y mantuvo el mismo número en el seguimiento. Finalmente, la participante 3, quien obtuvo 63 puntos en el pretratamiento, aumentó su puntuación a 81 en el postratamiento, y se mantuvo en el seguimiento con 80 puntos.

Figura 2. Niveles de atención plena.

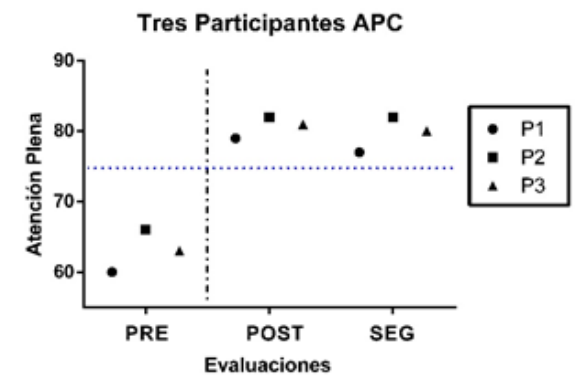


La Figura 3 muestra las puntuaciones en bruto de la escala ECOM. Se aprecia en ella que las participantes que asistieron al programa de APC aumentaron sus niveles en esta variable. La participante 1, de tener 66 puntos en el pretratamiento, aumentó a 84 en el postratamiento y disminuyó a 82 en el seguimiento. La participante 2 obtuvo 70 puntos en el pretratamiento, alcanzó 91 en el postratamiento y 90 en el seguimiento. Por último, la participante 3 obtuvo 66 puntos en el pretratamiento, aumentó en el postratamiento a 90 y se mantuvo en el seguimiento con 88 puntos.

Figura 3. Niveles de compasión.

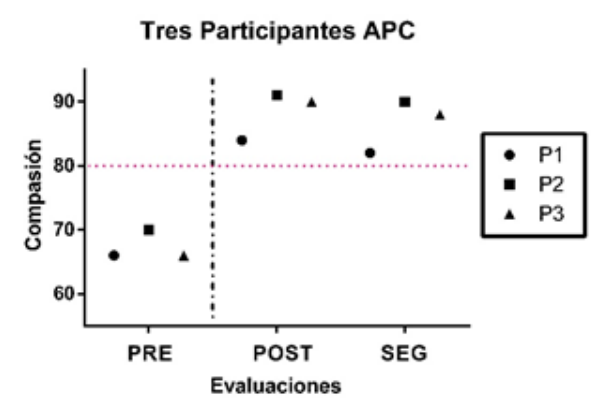

La Tabla 3 analiza el CCO en los porcentajes obtenidos en las variables de atención plena y compasión. Las tres mujeres que asistieron al modelo de APC aumentaron sus porcentajes en el postratamiento y obtuvieron una mejora clínica significativa en dichas variables, resultados que se mantuvieron en el seguimiento.

Tabla 3. CCO en atención plena y compasión.

\begin{tabular}{|c|c|c|c|c|c|}
\hline \multirow{2}{*}{ Tratamiento } & \multirow{2}{*}{ Participantes } & \multicolumn{2}{|c|}{ Atención plena } & \multicolumn{2}{c|}{ Compasión } \\
\cline { 3 - 6 } & & post-pre/pre & seg-pre/pre & post-pre/pre & seg-pre/pre \\
\hline \multirow{3}{*}{ APC } & P1 & $31 \% *$ & $28 \% *$ & $27 \% *$ & $24 \% *$ \\
\cline { 2 - 6 } & P2 & $24 \% *$ & $24 \% *$ & $30 \% *$ & $28 \% *$ \\
\cline { 2 - 6 } & P3 & $28 \% *$ & $26 \% *$ & $36 \% *$ & $36 \% *$ \\
\hline
\end{tabular}

* Cambio clínicamente significativo cuando es mayor a 20\%

\section{DISCUSIÓN}

En esta investigación se pudo observar que tanto la terapia cognitivo-conductual como la de atención plena y compasión lograron favorecer la disminución de sintomatología ansiosa en las seis mujeres embarazadas que concluyeron la intervención. Sin embargo, en los seguimientos a tres meses se pudo notar una tendencia a mantener una mayor reducción de la ansiedad en las participantes en el programa de APC. Ambos tratamientos pueden considerarse benéficos para mujeres embarazadas con características similares a las que participaron en esta investigación.

Además, este hallazgo concuerda con estudios preliminares, donde tanto la TCC (Green et al., 2015) como la APC (Matvienko-Sikar et al., 2016) lograron cambios clínicos en mujeres em- barazadas con sintomatología ansiosa. Asimismo, las participantes que recibieron el modelo de APC lograron aumentar sus niveles de atención plena y de compasión. Estudios como el de Goodman et al. (2014), corroboran los beneficios del entrenamiento en atención plena al incidir en estados afectivos positivos, así como en la disminución de niveles de estrés y ansiedad en mujeres embarazadas.

Entre las limitaciones del presente trabajo puede cuestionarse que el tamaño de la muestra fue demasiado pequeño, aunque esto se debió a las dificultades que tuvieron las participantes para asistir o continuar con los tratamientos psicológicos. Sin embargo, en estudios posteriores sería conveniente aumentar el tamaño de la muestra para lograr una mayor validez externa, así como generar grupos controles o de comparación para aumentar la validez interna. Se propone que en 
futuras investigaciones se indague en variables tales como los indicadores fisiológicos asociados a la respuesta de estrés-ansiedad, la adherencia al tratamiento y la evaluación de los componentes de cada modelo de intervención.

Esta investigación preliminar permitió implementar dos servicios de salud mental de bajo costo y alta eficacia para reducir la sintomatología ansiosa en mujeres mexicanas embarazadas. Por lo anterior, sería conveniente que estos dos modelos de tratamiento continúen siendo probados para que en un futuro puedan ser proporcionados a un mayor número de mujeres a fin de que disminuyan los riesgos asociados a la ansiedad durante el embarazo.

\section{REFERENCIAS}

Aceves K., G.S., Ureña B., E.C., Contreras M., E.D., Magaña M., A.S. y Romero M., A.R. (2013). Ansiedad y depresión en pacientes embarazadas. Atención Familiar, 20(1), 25-27. Doi: 10.1016/S1405-8871(16)30082-7.

Baer, R.A. (2011). Measuring mindfulness. Contemporary Buddhism, 12(1), 241-261. Doi: 10.1080/14639947.2011.564842.

Barlow, D.H. (2009). Single case experimental designs: Strategies for studyng behavior change. New York: Pearson.

Barrondo S., M., Martínez P., A.S. y González M., P. G.-P. (2008). Trastornos de ansiedad en atención primaria. Jano: Medicina y Humanidades, 1714, 9-14.

Beck, A.T. (2005). The current state of cognitive therapy: A 40-year retrospective. Archives of General Psychiatry, 62(9), 953-959. Doi: 10.1001/archpsyc.62.9.953.

Borkovec, T.D. (2006). Applied relaxation and cognitive therapy for pathological worry and generalized anxiety disorder. En C. L. Graham Davey y A. Wells (Eds.): Worry and its psychological disorders: Theory, assessment and treatment (pp. 273-287). New York: John Wiley \& Sons Ltd.

Bornemann, B. y Singer, T. (2013). What do we (not) mean by training. En T. Singer y M. Bolz (Eds.): Compassion. Bridging practice and science. Leipzig (Alemania): Max Planck Society.

Brown, K.W. y Ryan, R.M. (2003). The benefits of being present: Mindfulness and its role in psychological well-being. Journal of Personality and Social Psychology, 84(4), 822-848.

Cano V., A., Dongil C., E., Salguero, J.M. y Wood, C.M. (2013). Intervención cognitivo-conductual en los trastornos de ansiedad: una actualización. Información Psicológica, 102, 4-27.

Cardiel R., M. (1994). La medición de la calidad de vida. En L. Moreno, F. Cano y H. García (Eds.): Epidemiología clínica (pp. 189-199). México: Interamericana-McGraw-Hill.

Clark, D. y Beck, A. (2013). Terapia cognitiva para trastornos de ansiedad. Bilbao (España): Descleé de Brouwer.

Díaz, M., Ruiz, M.Á. y Villalobos, A. (2017). Manual de técnicas y terapias cognitivo-conductuales. Bilbao (España): Desclée de Brouwer.

Ding, X.-X., Wu Y.-L., Xu, S.-J., Zhu, R.-P., Jia, X.-M., Zhang, S.-F., Huang, K., Zhu, P., Hao, J.-H. y Tao, F.-B. (2014). Maternal anxiety during pregnancy and adverse birth outcomes: A systematic review and meta-analysis of prospective cohort studies. Journal of Affective Disorders, 159, 103-110.

Epp, A., Dobson, K. y Cottraux, J. (2009). Applications of Individual cognitive-behavioral therapy to specific disorders efficacy and indications. En G. O. Gabbard (Ed.): Textbook of psychotherapeutic treatments. American Psychiatric Association.

Fairbrother, N., Janssen, P., Antony, M.M., Tucker, E. y Young, A.H. (2016). Perinatal anxiety disorder prevalence and incidence. Journal of Affective Disorders, 200, 148-155.

Glover, V., Ahmed-Salim, Y. y Capron, L. (2016). Maternal anxiety, depression, and stress during pregnancy: Effects on the fetus and the child, and underlying mechanisms. En N. Reissland y B. S. Kisilevsky (Eds.): Fetal development (pp. 213-227). New York: Springer.

Goldin, P.R. y Gross, J.J. (2010). Effects of mindfulness-based stress reduction (MBSR) on emotion regulation in social anxiety disorder. Emotion, 10(1), 83-91. Doi: 10.1037/a0018441.

Goodman, J.H., Guarino, A., Chenausky, K., Klein, L., Prager, J., Petersen, R., Forget, A. y Freeman, M. (2014). Calm Pregnancy: Results of a pilot study of mindfulness-based cognitive therapy for perinatal anxiety. Archives of Women's Mental Health, 17(5), 373-387.

Green, S.M., Haber, E., Frey, B.N. y McCabe, R.E. (2015). Cognitive-behavioral group treatment for perinatal anxiety: A pilot study. Archives of Women's Mental Health, 18(4), 631-638.

Guardino, C.M., Dunkel-Schetter, C., Bower, J.E., Lu, M.C. y Smalley, S.L. (2014). Randomised controlled pilot trial of mindfulness training for stress reduction during pregnancy. Psychology \& Health, 29(3), 334-349. 
Hangartner, D. (2013). Human suffering and the four immeasurables: A Buddhist perspective on compassion. En T. Singer y M. Bolz (Eds.): Compassion: bridging practice and science (pp. 152-164). Munich: Max Planck Society.

Helmes, E. y Ward, B.G. (2017). Mindfulness-based cognitive therapy for anxiety symptoms in older adults in residential care. Aging \& Mental Health, 21(3), 272-278.

Hernández, R., Fernández, C. y Baptista, P. (2003). Metodología de la investigación. México: McGraw-Hill.

Hoehn-Saric, R. (1998). Psychic and somatic anxiety: Worries, somatic symptoms and physiological changes. Acta Psychiatrica Scandinavica, 98(s393), 32-38.

Hoffman, C.J., Ersser, S.J., Hopkinson, J.B., Nicholls, P.G., Harrington, J.E. y Thomas, P.W. (2012). Effectiveness of mindfulness-based stress reduction in mood, breast-and endocrine-related quality of life, and well-being in stage 0 to III breast cancer: A randomized, controlled trial. Journal of Clinical Oncology, 30(12), 1335-1342.

Jurado, S. (en prensa). Inventario Mexicano de Ansiedad, Depresión e Ideación Suicida Versión Beta. México: UNAM.

Kingdon, D. y Dimech, A. (2008). Cognitive and behavioural therapies: The state of the art. Psychiatry, 7(5), 217-220.

Koszycki, D., Thake, J., Mavounza, C., Daoust, J.-P., Taljaard, M. y Bradwejn, J. (2016). Preliminary investigation of a mindfulness-based intervention for social anxiety disorder that integrates compassion meditation and mindful exposure. The Journal of Alternative and Complementary Medicine, 22(5), 363-374.

Lemon, E.L., Vanderkruik, R. y Dimidjian, S. (2015). Treatment of anxiety during pregnancy: Room to grow. Archives of Women's Mental Health, 18(3), 569-570. Doi: 10.1007/s00737-015-0514-3.

Leonetti, L. y Martins, M. (2007). Ansiedad maternal en el periodo prenatal y postnatal: Revisión de la literatura. Revista Latino-Americana Enfermagem, 15(4), 677-683.

López M., E., Hernández P., M.R., Méndez S., L., Gutiérrez G., J.J., Araujo D., D., Nuñez G., A., Cervantes S., L.K., Nava A., S., Bautista G., L.E. y Hölzel, B.K. (2015). Psychometric properties of the Mexican version of the mindful attention awareness scale (MAAS). Psychologia. Avances de la Disciplina, 9(1), 13-27.

Marchesi, C., Ossola, P., Amerio, A., Daniel, B.D., Tonna, M. y De Panfilis, C. (2016). Clinical management of perinatal anxiety disorders: A systematic review. Journal of Affective Disorders, 190, 543-550.

Matvienko-Sikar, K., Lee, L., Murphy, G. y Murphy, L. (2016). The effects of mindfulness interventions on prenatal well-being: A systematic review. Psychology \& Health, 31(12), 1415-1434.

Miró B., M.T. (2012). Mindfulness y auto-observación terapéutica. En M. T. Miró B. (Ed.): Mindfulness en la práctica clínica (pp. 15-50). Bilbao (España) Desclée de Brouwer.

O’Leary, K., Dockray, S. y Hammond, S. (2016). Positive prenatal well-being: Conceptualising and measuring mindfulness and gratitude in pregnancy. Archives of Women's Mental Health, 19(4), 665-673.

Paquin E., M.S. y Coutiño A., M. (2017). Efectos del tratamiento de atención plena y compasión sobre ansiedad, calidad de vida y asma. Psicología y Salud, 27(2), 167-178.

Pereira A., T., Xavier, S., Bento, E., Azevedo, J., Marques, M., Soares, M.J., Freitas, V., Pinto, A.M., Roque, C. y Macedo, A. (2016). Mindfulness, self-compassion and depressive symptoms in pregnant women. European Psychiatry, 33, S420.

Pérez Á., M. (2012). Third-generation therapies: Achievements and challenges. International Journal of Clinical and Health Psychology, 12(2), 291-310.

Pimentel N., D., Morales C., F., Sánchez B., C. y Meza R., P. (2013). Intervención psicológica institucional oportuna en pacientes embarazadas con diabetes mellitus. Perinatología y Reproducción Humana, 27(2), 98-105.

Reck, C., Zimmer, K., Dubber, S., Zipser, B., Schlehe, B. y Gawlik, S. (2013). The influence of general anxiety and childbirth-specific anxiety on birth outcome. Archives of Women's Mental Health, 16(5), 363-369.

Ricard, M. (2009). El arte de la meditación. Barcelona: Urano, S.A.

Rygh, J.L. y Sanderson, W.C. (2004). Treating generalized anxiety disorder: Evidence-based strategies, tools, and techniques. New York: The Guilford Press.

Sierra, J.C., Ortega, V. y Zubeidat, I. (2003). Ansiedad, angustia y estrés: tres conceptos a diferenciar. Revista Mal-estar e Subjetividade, 3(1), 11-60.

Silva M., M.J., Nogueira D., A., Clapis M., J. y Leite E., P.R.C. (2017). Anxiety in pregnancy: Prevalence and associated factors. Revista da Escola de Enfermagem da USP, 51.

Simón, V. y Germer, C. (2011). Aprender a practicar mindfulness. Barcelona: Sello Editorial.

Tello A., L. y Coutiño A., B.M. (2019). Escala de Compasión (ECOM) para población mexicana. Psicología y Salud, 29(1), 25-32.

World Health Organization (2020). Mental health. Facts y statistics. Geneve: WHO. 